\title{
3-Dimensional QR Code Generator for Improving Storage Capacity
}

\author{
Naresh Eeni ${ }^{1}$, Mahesh Gudivaka ${ }^{2}$ \\ M. Tech Student, Department of ECE, Bhimavaram Institute of Engineering and Technology, A.P, India ${ }^{1}$ \\ Assistant Professor, Department of ECE, Bhimavaram Institute of Engineering and Technology, A.P, India ${ }^{2}$
}

\begin{abstract}
QR code system is presently most popular encoding system. A QR code encodes the data by standardized encoding modes for storing the data efficiently. In this paper, a new 3-dimensional QR code generator for improving storage capacity of the encoded data is proposed. This 3-dimensional QR(Quick Response) code generator encodes three different data vectors simultaneously. When compared to the traditional greyscale QR code, the proposed QR code increases the storage capacity. In this method, for each data set a greyscale QR code is generated such a way that the three greyscale QR codes are generated. By combining these three greyscale QR codes, a new 3-dimensional QR code is generated.
\end{abstract}

Keywords: QR code, Storage capacity.

\section{INTRODUCTION}

A barcode is an optical machine readable representation of data, which shows data about the object to which it attaches. Barcode is predefined format of dark bars and white spaces. It allows real time data to be collected accurately and rapidly. Instead of manually typing a customer identification number into a database, if the information is contained in a barcode a data entry operator may scan it. It reduces human error.

But barcodes have some disadvantages like less amount of data can be stores and the data is present only in the horizontal direction. The barcode recognition module consists of three parts: bar detection, barcode detection, and a barcode comparison block. The bar detection block detects bars from the barcode feature signal.

First, it tries to identify a black bar, if it is not there, then the first bar has zero width. If there is a black bar, then it calculates the pixels of the black bar. For the white bars, it does the same. After the bar detections, the bar code detection begins with the beginning bars and calculates all the possible values of barcode values that may form a valid string with all the possible separators.

This function returns sequence of indice stobar code guard bars. The bar code comparison block takes in the code book for all the encoded GTIN13 barcode values. It also reverses it for determining the last 6 digits of the GTIN13 barcode. The barcode recognition block takes in the barcodes and tries to matchup the barcode with the numbers of pixels generated from the bar detection.

In order to ensure better accuracy, thev alues are calculated from the left to right and right to left. The normalized confidence is calculated. The barcode recognition block set returns the barcode and the normalized confidence. In order to overcome these disadvantages, a new code format is introduced. QR Code is two-dimensional barcode which is categorized in matrix barcode that can store data information.

QR stands for "Quick Response" as the creator intended the code to allow its contents to be decoded at high speed. It is introduced in Japan by Denso Corporation in 1994. This kind of barcode was initially used for tracking inventory in vehicle parts manufacturing and is now used in a variety of industries. Nowadays, mobile phones with built-in camera are wildly used to recognize the QR code.

There have been many URL shortening services that automatically generate QR code links to websites such as Goo.gl and Bit.ly. Goo.gl is the first introduced URL shortening service that automatically generates QR codes. The URL shortening service shortens link and turn it into a QR code that, when scanned with a mobile QR code reader, automatically direct users to the shortened link.

It really shows that $\mathrm{QR}$ codes are going to become more and more popular. 


\section{3-DIMENSIONAL QR CODE}

The traditional QR encoding converts text into single image plane such coding has less storage space. The proposed 3dimesional QR code has takes three text files for encoding each text file converts into QR code.

\section{RSA METHODOLOGY}

RSA (Rivest-shamir-Adleman) is a cryptosystem for public-key encryption and it is widely used for securing sensitive data, particularly when being sent over an insecure network such as the Internet.Public-key cryptography, also known as asymmetric cryptography, uses two different but mathematically linked keys. They are public key and private key. The public key can be shared with everyone, whereas the private key must be kept secret. In RSA cryptography, both the public and the private keys can encrypt a message; the opposite key from the one used to encrypt a message is used to decrypt it.

This attribute is one reason why RSA has become the most widely used asymmetric algorithm. It provides a method of assuring the authenticity, confidentiality, integrity and non-reputability of data storage and electronic communications. In this algorithm, different key is used on both sides. Private key is used on the encryption side and in the same way a public key is used in decryption side. In RSA algorithm, there are mainly three steps: key generation, encryption and decryption procedure.

\section{Key Generation:}

1. Select the two prime numbers $p, q$ and $p \neq q$.

2. Calculate $\mathrm{n}=\mathrm{p} \times \mathrm{q}$.

3. $\varphi(\mathrm{n})$ calculated as $\varphi(\mathrm{n})=(\mathrm{p}-1) \times(\mathrm{q}-1)$.

4. Select integer e whose $\operatorname{gcd}(\varphi(n), e)=1,1<e<n$.

5. Calculated such that Ed-1 is exactly divisible by $\varphi(n)$.

6. Public key $(e, n)$.

7. Private key $(\mathrm{d}, \mathrm{n})$

\section{Reed-Solomon code:}

Reed-Solomon codes are a group of error-correcting codes. It is able to detect and correct multiple symbol errors. In communication, there is a possibility of occurring either single bit or multiple bit error, but in data storage it is burst errors. So, it is desirable to use the Reed-Solomon codes in the data storage. The encoding process of RS code as follows.

- Multiply the non-binary message polynomial $\mathrm{m}(\mathrm{x})$ by $\mathrm{x}^{\mathrm{n}-\mathrm{k}}$

- Dividing $\mathrm{x}^{\mathrm{n}-\mathrm{k}} \mathrm{m}(\mathrm{x})$ by $\mathrm{g}(\mathrm{x})$ to obtain the remainder $\mathrm{b}(\mathrm{x})$

- Forming the codeword $\mathrm{b}(\mathrm{X})+\mathrm{x}^{\mathrm{n}-\mathrm{k}} \mathrm{u}(\mathrm{x})$

\section{IV.QR ENCODING ALGORITHM}

1. Enter the confidential information "My College is BIET" and generate the image and assign Red plane to this message.

2.Now, convert the above message into ASCII equivalent.

3.Apply the RSA algorithm for each value with Private key.

4.Enter another confidential information "M.Tech" and assign green plane to this message

5.Now, convert the above message into ASCII equivalent and apply RSA algorithm.

6.The same procedure is performed for another message "QR code" which was assigned by the blue plane.

7.Now, combine all the three planes.

8.Generate the codeword for the given information by using non-binary RS code.

9.Convert the codeword into binary and place these bits in QR pattern

10. Decoding process is carried out by following steps

Read the colour QR code.

Split into RGB planes.

Read the information bits from QR code.

Convert these bits into decimal and eliminate the parity bytes by using an RS decoding process.

Apply RSA algorithm by using Public key to get the original information.

Figure.1. shows the complete encoding procedure 


\section{IJARCCE}

International Journal of Advanced Research in Computer and Communication Engineering ISO 3297:2007 Certified

Vol. 6, Issue 4, April 2017

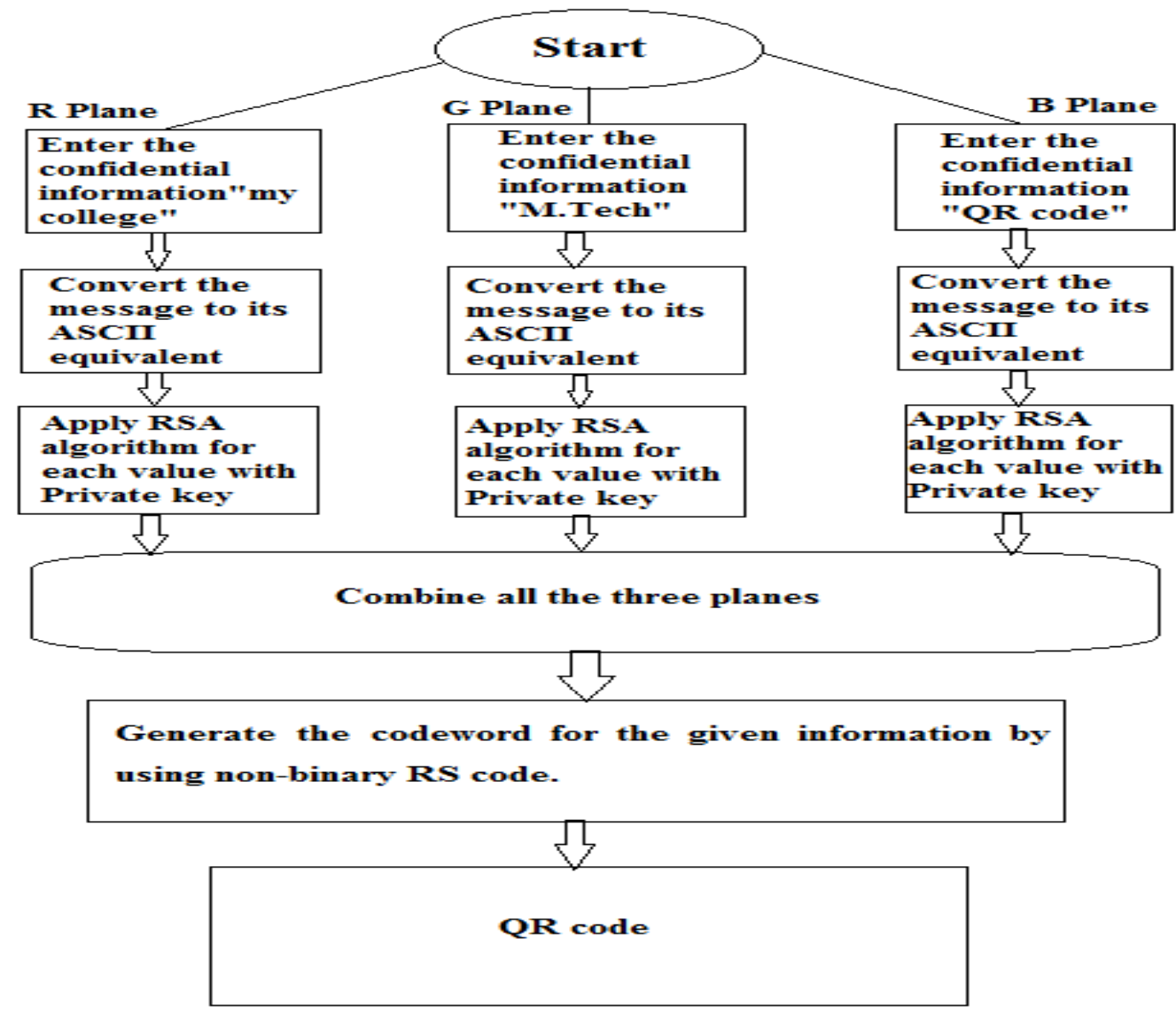

Fig.1.Flow chart of 3-dimensional QR Code

\section{SIMULATION RESULTS}

The encoding and decoding procedure of QR code is given as follows, initially three input messages for three different colour planes.

Message data $\mathrm{R}$ plane is: 'my college is biet'

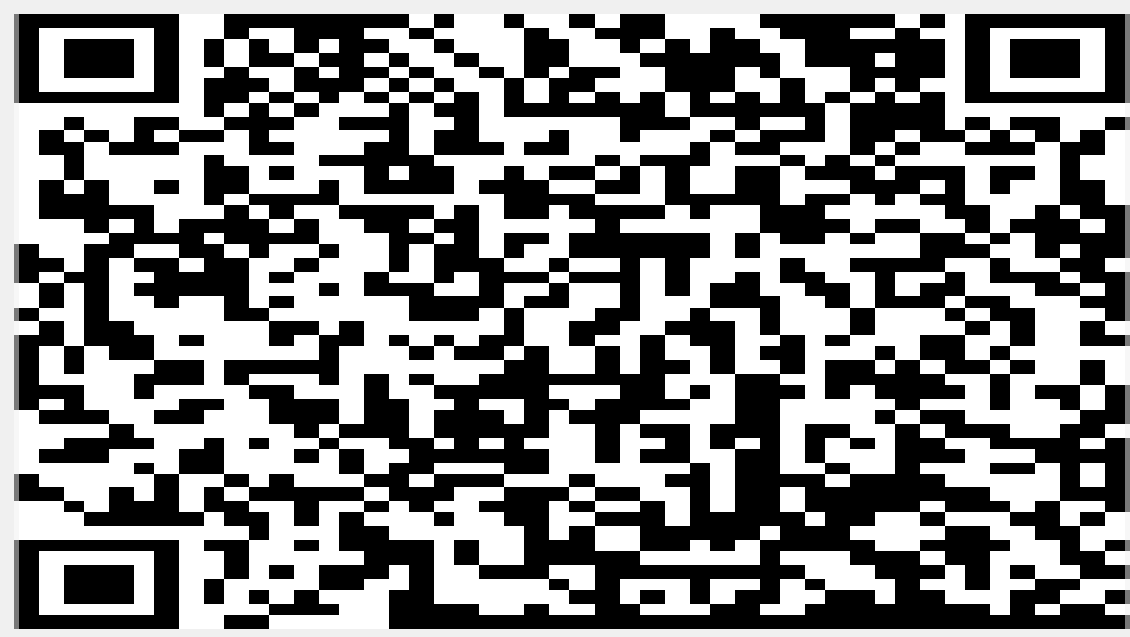

Fig.2.Red channel QR code

Message data $G$ plane is: 'i am doing M.Tech now' 
International Journal of Advanced Research in Computer and Communication Engineering ISO 3297:2007 Certified

Vol. 6, Issue 4, April 2017

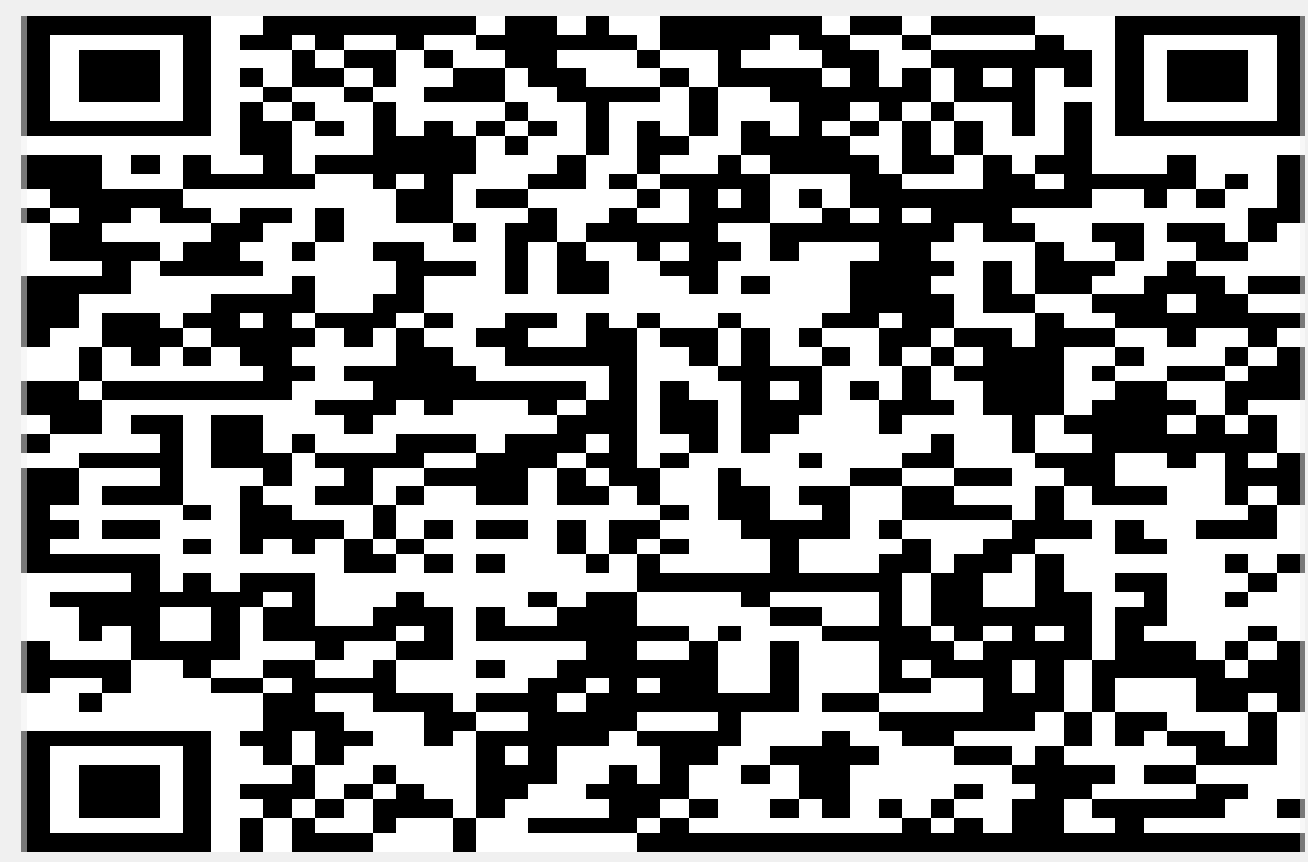

Fig.3. Green channel QR code

Message data B plane is: 'my project qr code'

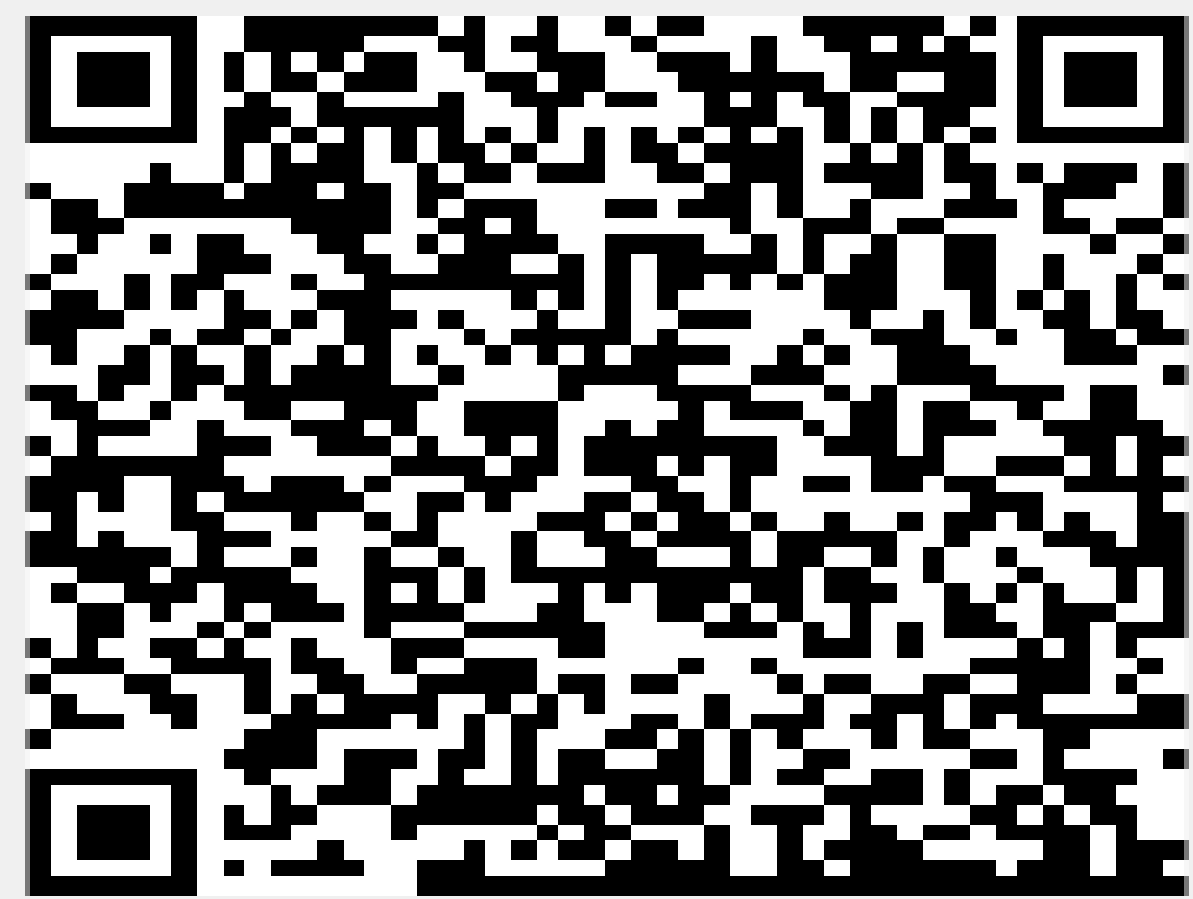

Fig.4. Blue channel QR code

By combining the three planes three dimensional $\mathrm{QR}$ code is formed 


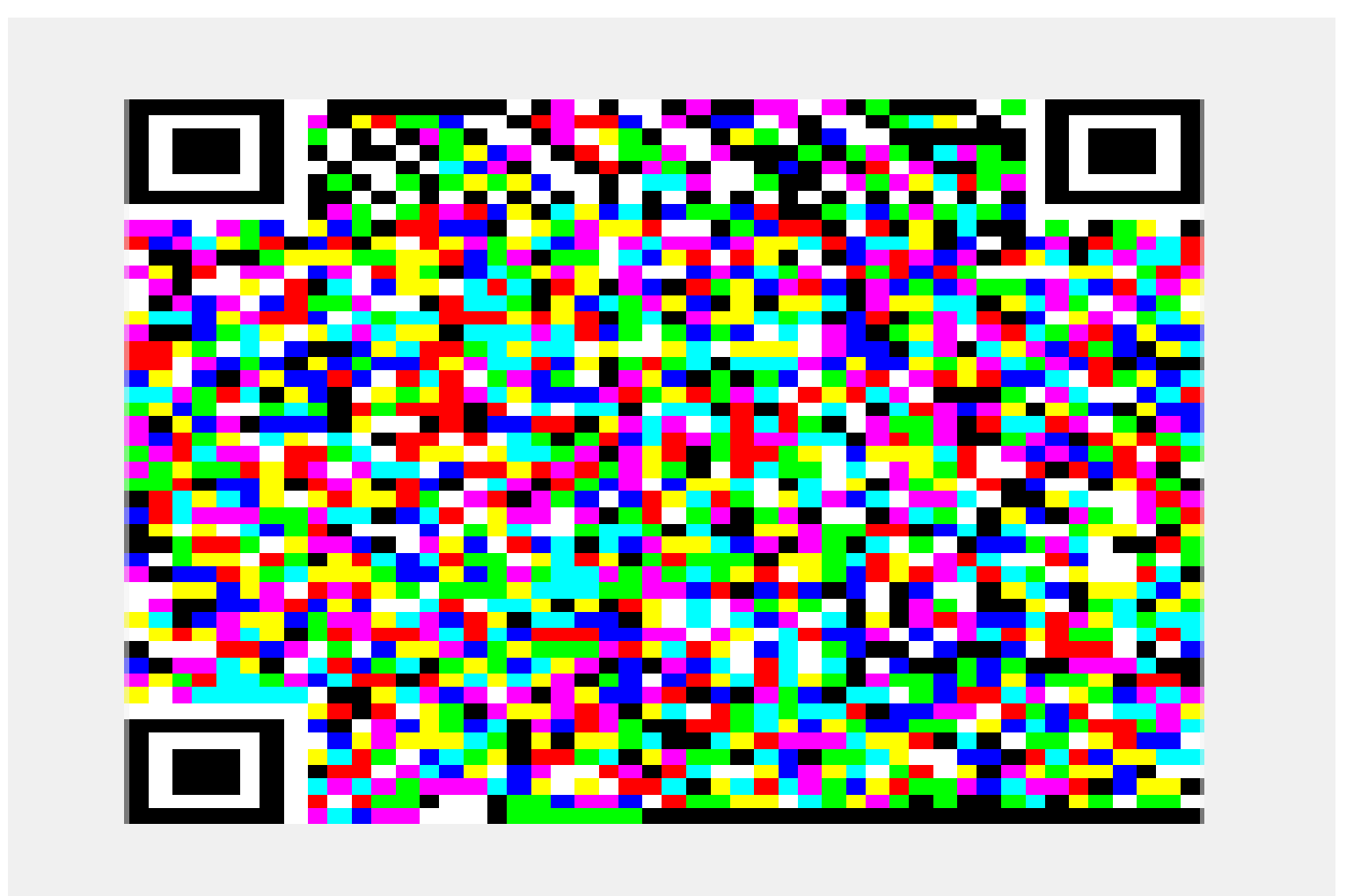

FIG.5. 3-D QR CODE

\section{CONCLUSION}

A new approach to $\mathrm{QR}$ code encoding is proposed in this paper which gives the better storage when compared to traditional QR code encoding. The RSA encryption methodology provides the additional security feature to the QR code the composition of three colour planes carry's more data compared to grey scale QR code. The proposed method will broaden the applications of QR code in the field of data transmission and security.

\section{REFERENCES}

[1]. Yue Liu, Ju Yang, Mingjun Liu, "Recognition of QR Code with mobile phones," Control and Decision Conference, CCDC 2008. Chinese, pp. 203 - 206, 2-4 July 2008.

[2]. Yu-HsuanChang, Chung-Hua Chu and Ming-Syan Chen, "A General Scheme for Extracting QR Code from a Non-uniform Background in Camera Phones and Applications," Ninth IEEE International Symposium on Multimedia, ISM 2007. pp. 123-130, 1012 Dec. 2007

[3]. Yuan-Cheng Lai, Frannie Han, Yi-HsuanYeh, Ching-Neng Lai and Yu-Chin Szu, "A GPS navigation system with QR code decoding and friend positioning in smart phones," 2nd International Conference on Education Technology and Computer (ICETC), pp.V5-66-V5-70, 2224 June 2010 .

[4]. Information technology - Automatic identification and data capture techniques — QR Code 2005 bar code symbology specification, 2006.

[5]. Tasos Falas, Hossein Kashani, "Two-Dimensional Bar-code Decoding with Camera-Equipped Mobile Phones," Proceedings of the Fifth Annual IEEE International Conference on Pervasive Computing and Communications Workshops (PerComW'07), 19-23, March, 597-600,2007.

[6]. Gao, Jerry Zeyu, et al, "Understanding 2D-BarCode Technology and Applications in M-Commerce," The 31st Annual International Computer Software and Applications Conference (COMPSAC 2007). Volume 2, July 24 - 27, 49 - 56, 2007.]

[7]. Eisaku Ohbuchi, Hiroshi Hanaizumi, and Lim Ah Hock, "Barcode Readers using the Camera Device in Mobile Phones," Proceedings of the 2004 International Conference on Cyberworlds (CW04), 11, 260-265,2004.

[8]. H. Kurosaka, K. Miyamoto, Y. Nagase, H. Ikegami, K. Sato, and M. Otsubo. Mobile Phone Application LSI Design Using C based Design Methodology. Technical Report 4, NEC Corporation, 42003. 